\title{
Area-Based Differentials in Childhood Cancer Incidence in Australia, 1996-2006
}

Danny R Youlden, BSc, ${ }^{1}$ Peter D Baade, PhD, ${ }^{1,2}$ Patricia C Valery, MD, MPH, PhD, ${ }^{3,4}$ Timothy E Hassall, MB BS, FRACP, ${ }^{5}$ Leisa J Ward, ${ }^{1}$ Adele C Green, MB BS, PhD, MSc, ${ }^{3}$ Joanne F Aitken, BSc, SM, $\mathrm{PhD}^{1,6}$

1. Viertel Centre for Research in Cancer Control, Cancer Council Queensland, 553 Gregory Terrace, Fortitude Valley Queensland 4006, Australia.

2. School of Public Health, Queensland University of Technology, Victoria Park Road, Kelvin Grove Queensland 4059, Australia.

3. Queensland Institute of Medical Research, 300 Herston Road, Herston Queensland 4006, Australia.

4. The Australian Centre for International and Tropical Health, University of Queensland, Herston Road, Herston Queensland 4006, Australia.

5. Royal Children’s Hospital, Herston Road, Herston Queensland 4006, Australia.

6. School of Population Health, University of Queensland, Herston Road, Herston Queensland 4006, Australia.

Corresponding author:

Danny Youlden

Viertel Centre for Research in Cancer Control

Cancer Council Queensland

PO Box 201

Spring Hill Queensland 4006, Australia

Fax: +61 732598527

Email: dannyyoulden@cancerqld.org.au

Key words or phrases: childhood cancer, incidence, remoteness, socioeconomic status, Australia.

Word count: Abstract - 242; Text - 2,420 (excluding tables and references)

Number of tables: 2

Number of figures: 0

Running title: Area-based differentials in childhood cancer 


\section{ABSTRACT}

Background: International studies examining the association between the incidence of childhood cancer and characteristics of the area in which the patient lives have generally reported inconsistent patterns. Area-based differentials in childhood cancer throughout Australia have not been previously published at a national level.

Procedure: Population-based information from the Australian Paediatric Cancer Registry was used to identify all children aged 0-14 years old diagnosed with invasive cancer or intracranial and intraspinal tumours of benign or uncertain behaviour between 1996-2006. Age-standardised incidence rates per million children per year and the corresponding incidence rate ratios were calculated, categorised by remoteness of residence and an area-based index of socioeconomic disadvantage. Results were also stratified by the most common types of childhood cancer. Results: There was a significant, decreasing gradient in the incidence of childhood cancer as remoteness of residence increased. Children living in remote or very remote areas were $21 \%$ less likely to be diagnosed with cancer compared to children in major cities, mainly due to differences in the incidence of leukaemias and lymphomas. This differential was no longer significant when only non-Indigenous children were considered. No clear relationship was found between incidence and socioeconomic status in contrast to similar earlier studies.

Conclusions: The findings by remoteness of residence are consistent with the lower incidence rates of cancer that are typically associated with Indigenous Australians. There is also a suggestion that the aetiological factors associated with childhood leukaemia and socioeconomic status may have altered over time. 


\section{INTRODUCTION}

Although cancer among children is relatively rare, the consequences are far-reaching. In particular, cancer is the most common cause of disease-related death among children aged 1-14 years old in both Australia[1] and other high income countries.[2] Beyond the loss of young lives, the burden of childhood cancer extends to the long-term adverse health effects that may be experienced by survivors, $[3,4]$ as well as significant psychosocial and financial imposts on their families.[5,6]

The causes of childhood cancer are largely unclear.[7] A better understanding of how incidence differs depending on the characteristics of the area where a child lives may help to unravel some of the aetiological factors that contribute to the development of cancer. Studies examining this issue have generally been inconclusive when considered collectively.[8-15] A review of the potential link between childhood leukaemia and socioeconomic status (SES)[8] found the association varied depending on the time that the study was conducted, the study design (areabased versus case-control) and the measure used to determine SES. The authors identified the need for contemporary analyses using area-based data to establish whether the relationship between higher SES and the increased incidence of childhood leukaemia that was previously reported in some countries was still present.[8]

A study conducted in the early 1980s in Queensland (Australia)[16] reported a significant correlation between area-level SES and incidence rates of acute lymphoblastic leukaemia among children. However, a similar relationship with SES was not present for other types of childhood cancer.[16] Building on this work, we have utilised the latest available data from the Australian 
Paediatric Cancer Registry (APCR) to investigate at a national level the association between different types of childhood cancer and an area-based measure of SES. In addition, we also examined whether there was a link between remoteness of residence and the incidence of childhood cancer.

\section{METHODS}

The APCR is one of only a few national, population-based childhood cancer registries in the world.[17] Details on every case of childhood cancer (aged 0-14 years at diagnosis) are collected with the assistance of each State and Territory cancer registry and all major paediatric hospitals in Australia. The diagnostic group for each child was allocated according to the third edition of the International Classification of Childhood Cancers (ICCC-3), which covers all invasive cancers and intracranial and intraspinal tumours of benign or uncertain behaviour.[18]

Almost all (99.7\%) eligible cases of childhood cancer in the APCR occurring during the period 1996-2006 were able to be assigned to remoteness and SES categories based on address at diagnosis. The unmatched cases $(\mathrm{n}=18)$ were removed from the analysis. Population counts by sex, age group, year and locality were obtained from the Australian Bureau of Statistics.[19]

Four categories of remoteness were specified based on the Australian Standard Geographical Classification (ASGC) Remoteness Areas[20]: “Major cities”, "Inner regional”, “Outer regional" and "Remote/very remote". The ASGC remoteness classification is a purely geographic measure without any direct consideration of socioeconomic status. Broadly speaking, inner regional areas are those on the fringes of major cities where residents may 
experience minor restrictions in access to some services. Outer regional areas are generally of a more rural nature, while people in remote/very remote localities are living hundreds, if not thousands, of kilometres away from major cities and the services that they provide.

Road distance measurements to the nearest service centres were used to determine the remoteness category for each locality. Service centres were split into five groupings based on population size, ranging from between 1,000-4,999 residents (Category E service centre) up to $>250,000$ residents (Category A service centre). The five distance measurements for an individual locality were each standardized to a ratio by dividing by the national mean for the corresponding service centre category and then added together to give a total. More remote localities were represented by a higher score.

In the absence of individual indicators of SES, an area-based measure called the Index of Relative Socioeconomic Disadvantage (IRSD)[21] was used. Derived from national census information, the IRSD takes into account factors including the percentage of people in a locality with low income, low educational attainment, and who are either unemployed or employed in relatively unskilled occupations. Localities were categorised as "Least disadvantaged" (quintile 5 for IRSD), “Middle SES” (quintiles 2-4) or “Most disadvantaged” (quintile 1).

Incidence rates directly age-standardised to the 2000 World Standard Population[22] were calculated for all cancers combined and for the three largest diagnostic groups (leukaemias, tumours of the central nervous system (CNS) and lymphomas). The remaining cancer types 
were aggregated into a group labeled "Other solid tumours" to enable sufficient numbers for analysis.

Poisson regression was used to estimate incidence rate ratios and 95\% confidence intervals, with the selected baseline categories for remoteness and SES being those with the highest number of cases i.e. "Major cities” and "Middle SES” respectively. Models included sex, age group at diagnosis and either remoteness or SES, all specified as categorical variables. The significance of the gradient across the variables of interest was investigated by rerunning the models and fitting remoteness or SES as continuous variables. An interaction term for remoteness and SES was included in a separate model, with the remoteness categories "Outer regional" and "Remote/very remote” aggregated to allow sufficient numbers for the model to converge. For each model, the response variable was the number of childhood cancer cases in each strata offset by the log of the corresponding population, while goodness of fit was assessed using a chisquared test.

Indigenous children (those who identify as being of Australian Aboriginal or Torres Strait Islander origin) are estimated to comprise just over $2 \%$ of the population aged under 15 years old in major cities compared to approximately 24\% in remote/very remote parts of Australia.[23] Maintenance of traditional culture is more common in isolated areas.[24] The health status of Indigenous children also differs to that of non-Indigenous children across a range of indicators.[24] In order to assess the impact that these factors might have on the results of our investigation, the regression analysis by remoteness was repeated for non-Indigenous children only. 
All analyses were conducted using STATA/SE version 11.1 for Windows. Approval for this work was obtained from the ethics committees of the Queensland Institute of Medical Research, the University of Queensland, the cancer registries in all Australian States and Territories, and all hospitals that contributed to the data collection.

\section{RESULTS}

A total of 6,757 Australian children who were eligible for inclusion in the study were diagnosed with cancer during the study period. Over half of these children were male (54\%), and the distribution of age at diagnosis was $46 \%$ aged $0-4$ years, 25\% aged 5-9 years and 29\% aged 1014 years. Approximately two-thirds of cases had leukaemias (33\%), tumours of the CNS (23\%) or lymphomas $(10 \%)$.

The age-standardised incidence rates of all childhood cancer combined varied from 159.9 cases per million children per year in major cities to 126.5 cases per million children per year in remote/very remote areas. There was a significant, decreasing gradient $\left(p_{\text {grad }}=0.002\right)$ in the incidence rate ratios as remoteness of residence increased, with children from remote or very remote areas being $21 \%$ less likely to be diagnosed with cancer compared to those in major cities (Table I). Significant gradients by remoteness of residence were also found within the diagnostic groups of leukaemias and lymphomas. There was no discernable pattern in the incidence rate ratios by remoteness for either tumours of the CNS or other solid tumours. 
The association between remoteness and childhood cancers appeared to be mainly driven by the lower incidence reported among children in remote/very remote areas. When the relevant models were rerun only including incidence and population data for children who were identified as being non-Indigenous $(\mathrm{n}=5,972)$, the resulting gradients for the adjusted incidence rate ratios by remoteness of residence for all childhood cancers combined $\left(p_{\text {grad }}=0.333\right)$, leukaemia $\left(p_{\text {grad }}=0.536\right)$ and lymphoma $\left(p_{\text {grad }}=0.081\right)$ were no longer significant.

As shown in Table II, the overall incidence rate of childhood cancer was slightly higher among children in the least socioeconomically disadvantaged areas (162.0 cases per million children per year) compared to those in the most disadvantaged areas (150.7 cases per million children per year). However the gradient for the adjusted incidence rate ratios was not statistically significant $\left(p_{\text {grad }}=0.081\right)$. A similar pattern across the SES categories was observed for the adjusted incidence rate ratios among children with leukaemias, lymphomas and tumours of the CNS, but the gradient was non-significant within each of these diagnostic groups. The analysis was repeated for the diagnostic subgroup of lymphoid leukaemias in an attempt to verify the results from the previous study in Queensland,[16] but the association with SES also failed to reach statistical significance $\left(p_{\text {grad }}=0.146\right)$. Applying a different index of SES that was based on education and occupation only (as suggested by Poole and colleagues[8]) yielded comparable, insignificant gradients in childhood cancer incidence (results not shown).

There was no statistically significant evidence of interaction between remoteness and SES for all childhood cancers combined $(p=0.185)$ or for any of the diagnostic groupings (leukaemias: $p=0.632$; lymphomas: $p=0.816$; tumours of the CNS: $p=0.398$; other solid tumours: $p=0.340$ ). 


\section{DISCUSSION}

This is the first time that patterns of childhood cancer incidence by either remoteness or areabased SES have been investigated at a national level for Australia. We found that children living in more urban areas had higher rates of cancer overall, particularly for leukaemias and lymphomas. In contrast, there was little evidence of any differentials related to area-level measures of SES.

Published data on childhood cancer incidence by remoteness of residence are generally scarce, with the exception of a series of papers on the geographical distribution of childhood cancer in North-West England.[12-14] McNally and colleagues reported higher incidence of acute lymphoblastic leukaemia in areas with higher population density.[12] No corresponding association was found for lymphomas, which also exhibited a significant gradient across the remoteness categories in our study. Consistent with our results, they reported no clear relationship with rurality or population density for tumours of the central nervous system.[14] Another paper, which primarily examined an infectious mechanism (known as population mixing) for acute lymphoblastic leukaemia in England and Wales, failed to establish an association between the incidence of ALL and population density.[15]

Some of the variation regarding the impact of remoteness on childhood cancer incidence may be due to inherent geographic differences between Australia and the United Kingdom. In Australia, there are often much longer travelling distances to larger towns and cities for people living in a rural or remote area, with considerable implications in terms of time, cost and access to services. 
Furthermore, in the United Kingdom rural areas tend to be associated with higher SES[25] while the converse is generally true for Australia.[26]

A higher incidence rate of lymphoma in major cities throughout Australia has been previously reported among all persons, including adults and children.[27] However, as opposed to the findings for childhood leukaemia, no significant differences were reported in the incidence rates across the various remoteness categories for all leukaemia patients throughout Australia.[27] The factors which contribute to the excess of lymphoma in more urban areas irrespective of age at diagnosis are yet to be identified.

One potential reason for the significant differential in all childhood cancers by remoteness is that remote/very remote areas typically contain a relatively high proportion of Indigenous residents, and it is known that cancer incidence tends to be lower within this segment of the Australian adult population.[28] This concept was supported by a reanalysis of the data for non-Indigenous children only, with the modified gradient by remoteness of residence no longer significant. Even if this proves to be a plausible explanation for some of the observed variation, it is currently not known why cancer incidence would be lower among Indigenous children.

Another possible consideration involves the difficulties presented by the distances to specialised health care. Families of cancer patients who live in very isolated areas of Australia are confronted with major challenges regarding access to diagnostic, treatment and support services.[29-31] This could result in some level of underdiagnosis of cancer among children 
from remote communities, although it should be noted that there is no specific evidence to support this hypothesis.

Area-based analyses from the 1980s or earlier from countries including Australia, the United States and the United Kingdom described a fairly consistent relationship between higher SES and an increased incidence of childhood leukaemia.[8] This effect seems to have diminished more recently[9] as supported by our results, adding further weight to the theory that there may have been a shift in the underlying aetiology of childhood leukaemia over time.[8] No other significant trends in the incidence of other types of childhood cancer were evident by SES in either Australia or a similar analysis conducted in Canada, except for a lower rate of carcinomas in the poorest quintile of the Canadian population which may have been caused by random variation.[10]

One of the strengths of this study is the complete coverage of children with cancer in Australia through the APCR, combined with high data quality.[17] The population-based design prevents the introduction of selection bias that can occur with clinical trials. While information was not available from the APCR on personal indicators of SES for the parents of children with cancer, other authors have suggested that the use of area-based data as a proxy for individual SES may be more meaningful when considering the health of children.[9] We were unable to analyse data for each of the ICCC-3 diagnostic groups separately, apart from the three most common types of childhood cancer, due to the relatively small number of cases in some cells, particularly remote/very remote areas. This potentially could have prevented us from discovering specific 
area-based associations for other diagnostic groups that were not apparent after the data were combined.

In conclusion, our findings suggest that in Australia there is a significant gradient in the incidence of childhood cancer by remoteness of residence, particularly for leukaemias and lymphomas. The incidence of these childhood cancers is higher in urban than rural areas, in contrast to the poorer health that is typically experienced in regional and remote areas of Australia.[27] It appears that much of this differential is due to the Indigenous population in remote parts of the country. Future work is planned to examine childhood cancer incidence by Indigenous status in detail. Consistent with recent international case-control studies, our study supports the hypothesis that the relationship between area-based measures of SES and childhood cancer incidence has also weakened. Further research is needed to determine whether, in an environment of lower incidence, survival outcomes for rural childhood cancer patients are equitable with their urban counterparts. 


\section{ACKNOWLEDGEMENTS}

The support and assistance of staff at all Australian State and Territory Cancer Registries, the Australian Institute of Health and Welfare and the Medical Records Department at each of the paediatric oncology treating hospitals throughout Australia is gratefully acknowledged.

\section{CONFLICT OF INTEREST STATEMENT}

All authors are members of the Working Committee for the Australian Paediatric Cancer

Registry. The Australian Paediatric Cancer Registry is solely funded by Cancer Council

Queensland. PCV was supported by a National Health and Medical Research Council Public Health Training Grant. 


\section{REFERENCES}

1. Australian Institute of Health and Welfare. A picture of Australia's children 2009. AIHW Cat No PHE 112. Canberra: AIHW; 2009. 224 p.

2. Kellie SJ, Howard SC. Global child health priorities: what role for paediatric oncologists? Eur J Cancer 2008:44(16):2388-2396.

3. Hudson MM, Mertens AC, Yasui Y, et al. Health status of adult long-term survivors of childhood cancer: A report from the Childhood Cancer Survivor study. JAMA 2003:290(12):1583-1592.

4. Landier W, Bhatia S. Cancer survivorship: a pediatric perspective. Oncologist 2008:13(11):1181-1192.

5. Patterson JM, Holm KE, Gurney JG. The impact of childhood cancer on the family: a qualitative analysis of strains, resources, and coping behaviors. Psychooncology 2004:13(6):390-407.

6. Heath JA, Lintuuran RM, Rigguto G, et al. Childhood cancer: its impact and financial costs for Australian families. Pediatr Hematol Oncol 2006:23(5):439-448.

7. Stiller CA. Epidemiology and genetics of childhood cancer. Oncogene 2004:23(38):64296444.

8. Poole C, Greenland S, Luetters C, et al. Socioeconomic status and childhood leukaemia: a review. Int J Epidemiol 2006:35(2):370-384.

9. Adam M, Rebholz CE, Egger M, et al. Childhood leukaemia and socioeconomic status: what is the evidence? Radiat Prot Dosimetry 2008:132(2):246-254. 
10. Mezei G, Borugian MJ, Spinelli JJ, et al. Socioeconomic status and childhood solid tumor and lymphoma incidence in Canada. Am J Epidemiol 2006:164(2):170-175.

11. Stiller CA, Marcos-Gragera R, Ardanaz E, et al. Geographical patterns of childhood cancer incidence in Europe, 1988-1997. Report from the Automated Childhood Cancer Information System project. Eur J Cancer 2006:42(13):1952-1960.

12. McNally RJ, Alston RD, Cairns DP, et al. Geographical and ecological analyses of childhood acute leukaemias and lymphomas in north-west England. Br J Haematol 2003:123(1):60-65.

13. McNally RJ, Alston RD, Cairns DP, et al. Geographical and ecological analyses of childhood Wilms' tumours and soft-tissue sarcomas in North West England. Eur J Cancer 2003:39(11):1586-1593.

14. McNally RJ, Alston RD, Eden TO, et al. Further clues concerning the aetiology of childhood central nervous system tumours. Eur J Cancer 2004:40(18):2766-2772.

15. Stiller CA, Kroll ME, Boyle PJ, Feng Z. Population mixing, socioeconomic status and incidence of childhood acute lymphoblastic leukaemia in England and Wales: analysis by census ward. Br J Cancer 2008:98(5):1006-1011.

16. McWhirter WR. The relationship of incidence of childhood lymphoblastic leukaemia to social class. Br J Cancer 1982:46(4):640-645.

17. Baade PD, Youlden DR, Valery PC, et al. Trends in incidence of childhood cancer in Australia, 1983-2006. Br J Cancer 2010:102(3):620-626.

18. Steliarova-Foucher E, Stiller C, Lacour B, Kaatsch P. International Classification of Childhood Cancer, third edition. Cancer 2005:103(7):1457-1467. 
19. Australian Bureau of Statistics. Population by age and sex, Australian States and Territories, June 2008. ABS Cat No 3201.0. Canberra: ABS; 2008.

20. Australian Institute of Health and Welfare. Rural, regional and remote health: A guide to remoteness classifications. AIHW Cat No PHE 53. Canberra: AIHW; 2004. 77 p.

21. Australian Bureau of Statistics. Information paper: An introduction to Socio-Economic Indexes for Areas (SEIFA), 2006. ABS Cat No 2039.0. Canberra: ABS; 2008. 51 p.

22. Ahmad O, Boschi-Pinto C, Lopez A, et al. Age standardization of rates: a new WHO standard. GPE Discussion Paper Series No 31. Geneva: World Health Organization; 2001.

23. Australian Bureau of Statistics. Experimental Estimates and Projections, Aboriginal and Torres Strait Islander Australians, 1991 to 2021. ABS Cat No 3238.0. Canberra: ABS; 2009. $76 \mathrm{p}$.

24. Australian Institute of Health and Welfare and Australian Bureau of Statistics. The Health and Welfare of Australia's Aboriginal and Torres Strait Islander Peoples, 2008. AIHW Cat No IHW 21. Canberra: AIHW; 2008. 291 p.

25. Hays RB. Rural medical education in Europe: the relevance of the Australian experience. Rural Remote Health 2007:7(1):683.

26. Australian Bureau of Statistics. Australian social trends, 2000. ABS Cat No 4102.0. Canberra: ABS; 2000. 203 p.

27. Australian Institute of Health and Welfare. Rural, regional and remote health: Indicators of health status and determinants of health. AIHW Cat No PHE 97. Canberra: AIHW; 2008. $144 \mathrm{p}$.

28. Cunningham J, Rumbold AR, Zhang X, Condon JR. Incidence, aetiology, and outcomes of cancer in Indigenous peoples in Australia. Lancet Oncol 2008:9(6):585-595. 
29. Jong KE, Vale PJ, Armstrong BK. Rural inequalities in cancer care and outcome. Med J Aust 2005:182(1):13-14.

30. Bensink M, Wootton R, Irving $H$, et al. Investigating the cost-effectiveness of videotelephone based support for newly diagnosed paediatric oncology patients and their families: design of a randomised controlled trial. BMC Health Services Res 2007:7:38.

31. Heathcote K, Armstrong B. Disparities in cancer outcomes in regional and rural Australia. Cancer Forum 2007:31(2):70-74. 